\title{
Review of COVID-19 Vaccines Approved in the United States of America for Emergency Use
}

\author{
Deepa Vasireddy ${ }^{\mathrm{a}, \mathrm{f}}$, Paavani Atluri ${ }^{\mathrm{b}}$, Srikrishna Varun Malayalac, \\ Rachana Vanaparthy ${ }^{\mathrm{d}}$, Gisha Mohan ${ }^{\mathrm{e}}$
}

\begin{abstract}
Severe acute respiratory syndrome coronavirus 2 (SARS-CoV-2) is a novel coronavirus causing a global pandemic. Coronaviruses are a large family of single-stranded ribonucleic acid (RNA) viruses. The virus has four essential structural proteins which include the spike (S) glycoprotein, matrix $(\mathrm{M})$ protein, nucleocapsid $(\mathrm{N})$ protein and small envelope (E) protein. Different technologies are being used for vaccine development to battle the pandemic. There are messenger ribonucleic acid (mRNA)-based vaccines, deoxyribonucleic acid (DNA) vaccines, inactivated viral vaccines, live attenuated vaccines, protein subunit-based vaccines, viral vector-based vaccines and viruslike particle-based vaccines. Vaccine development has five stages. In the clinical developmental stage, vaccine development can be sped up by combining phase 1 and 2 . The vaccines can also be approved more swiftly on an emergent basis and released sooner for usage. The United States Food and Drug Administration (USFDA) has approved Pfizer-BioNTech, Moderna and Janssen coronavirus disease 2019 (COVID-19) vaccines for emergency use. There are other vaccines that have been approved around the world. The mRNA vaccines have been created using a novel technology and they contain a synthetically created RNA sequence of virus fragments encoding the S-protein which is injected. These vaccines have a relatively low cost of production and faster manufacturing time but can have comparatively lower immunogenicity and more than one dose of vaccine may be required. In the case of viral vector-based vaccines, genes encoding the SARS-CoV-2 S protein are isolated and following gene sequencings are introduced into the adenovirus vector. These vaccines have a relatively fast manufacturing time but the efficacy of the vaccine is variable based on the host's immune response to the viral vector. At the time of this paper, there were 81 vaccines in clinical development
\end{abstract}

Manuscript submitted March 23, 2021, accepted April 7, 2021

Published online April 27, 2021

aDepartment of Pediatrics, Pediatric Group of Acadiana, Lafayette, LA, USA bBay Area Hospital, Coos Bay, OR, USA

'Department of Medicine, Jeanes Campus - Temple University Hospital, Philadelphia, PA, USA

${ }^{\mathrm{d} O r e g o n ~ H e a l t h ~ a n d ~ S c i e n c e ~ U n i v e r s i t y, ~ P o r t l a n d, ~ O R, ~ U S A ~}$

ePhysicians for American Health Care Access, Philadelphia, PA, USA

fCorresponding Author: Deepa Vasireddy, Department of Pediatrics, Pediatric

Group of Acadiana, 7053 Johnston Street, Lafayette, LA 70503, USA.

Email: deepa26vasi@gmail.com

doi: https://doi.org/10.14740/jocmr4490 stage and 182 vaccines in preclinical development stage. Vaccines are an essential tool in our battle against COVID-19. Some of the COVID-19 vaccines have completed their phase III trials while many other potential vaccines are still in developmental stages. It used to take close to a decade for a vaccine to be developed and undergo rigorous testing until its production and availability to the public, but over the past year, we have seen multiple vaccines in different phases of testing against SARS-CoV-2 virus.

Keywords: COVID-19; SARS-CoV-2; Vaccines; Pfizer-BioNTech; Moderna; Janssen

\section{Introduction}

\section{Viral structure}

Severe acute respiratory syndrome coronavirus 2 (SARSCoV-2) is a novel coronavirus causing a global pandemic. Coronaviruses are a large family of single-stranded ribonucleic acid (RNA) viruses. The SARS-CoV-2 virus belongs to the Orthocoronovirinae subfamily and is classified as a betacoronavirus. It consists of positive sense non-segmented RNA held within an envelope. The virus has four essential structural proteins which include the spike (S) glycoprotein, matrix (M) protein, nucleocapsid (N) protein and small envelope (E) protein [1] (Fig. 1). Under the electron microscope, the virus appears spherical with a diameter that ranges from 60 to 140 $\mathrm{nm}$ and the outer surface is studded with spikes that are $9-12$ $\mathrm{nm}$ long [2] (Fig. 1). The spikes give it the appearance of a solar corona. The genome of the virus is about $30 \mathrm{~kb}$. It has a 5'cap structure and a 3'poly(A) tail. RNA replication and transcription requiring multi-stem loop structures are found at the 5' end. The four main structural proteins are encoded at the 3' end of the genome [3]. The $\mathrm{N}$ protein makes up the nucleocapsid by binding to the CoV RNA genome. It is considered to play a role in viral replication, transcription and translation. The protein has strong antigenicity and evokes an immunoglobulin G (IgG) driven antibody response to the nucleocapsid [4]. The E protein is an integral membrane protein that allows it to maintain the shape of the viral envelope and the protein also appears to have a role in viral propagation and pathogenicity in the host cells. E protein is now being studied 


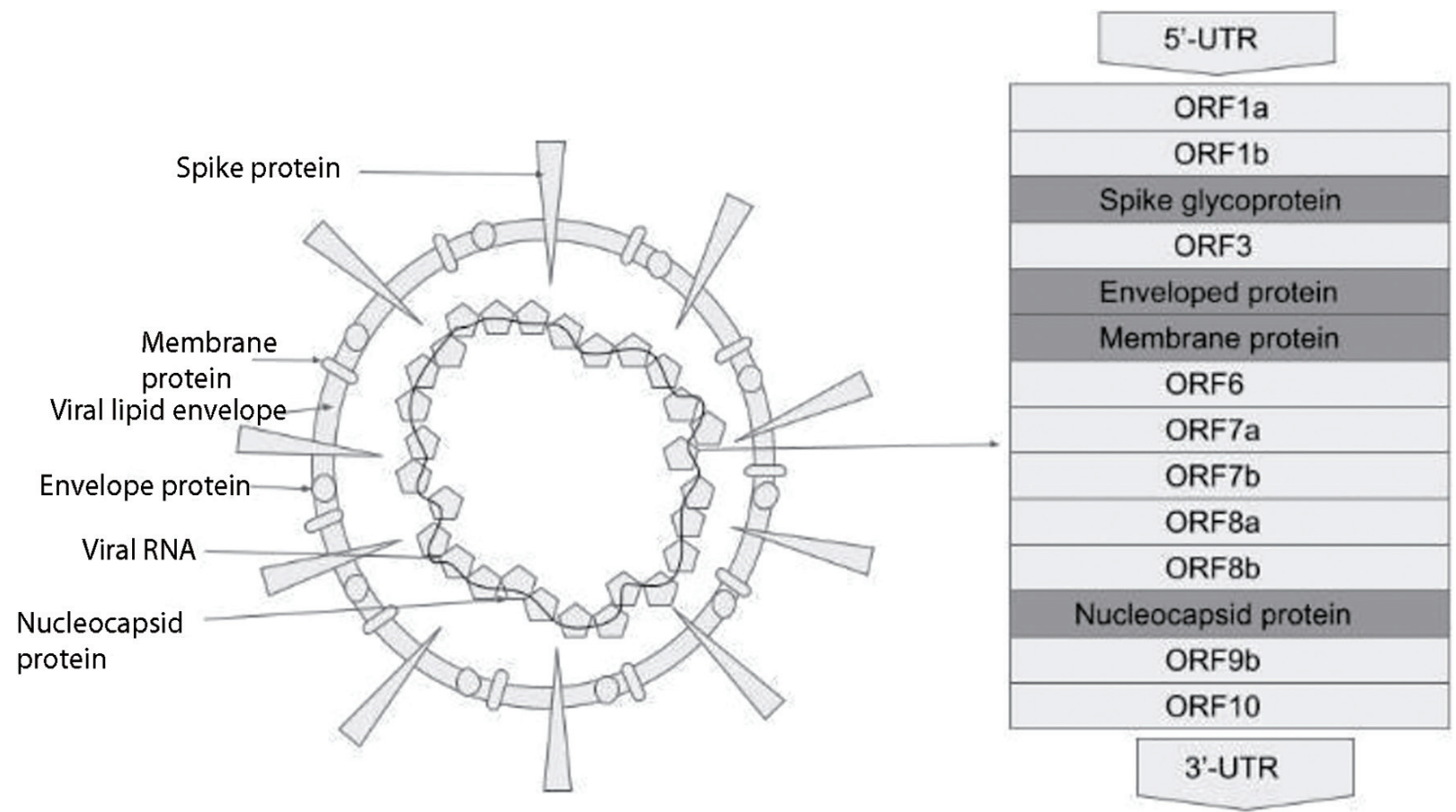

Figure 1. COVID-19 viral structure. COVID-19: coronavirus disease 2019.

for having potential ion channel properties which could be additive to or causative of its ability to interact with viral and host cell proteins, making it likely for it to have molecular functionality [5]. The M protein is a structural protein and can bind to the other proteins, thus assisting in viral assembly. It also, along with the $\mathrm{S}$ protein, seems to play a role in allowing the virus to attach to the host cell and enter into it [6]. The $\mathrm{S}$ glycoprotein is a homotrimeric class I fusion protein that mediates the host cell entry of the coronavirus. The S glycoprotein has two functional subunits that allow it to bind to the host cell receptor angiotensin-converting enzyme 2 (ACE2) (S1 subunit) and carry out the fusion between the viral and host cell membranes (S2 subunit) [7].

\section{Pathogenesis}

The initial process of the SARS-CoV-2 infection is the binding of the S protein with the ACE2 receptor which is followed by the invasion of the host cell that is triggered by proteases in the host cell. The viral RNA is then released into the host cell and gets translated. Non-structural proteins are formed post-translation and they form the complex that allows viral replication and transcription. During transcription, the viral proteins and genomic RNA are produced that are assembled into virions in the Golgi and endoplasmic reticulum and are then released out the host cell in vesicles [8]. Once the virions are released, the host's innate immune system made up of $\mathrm{T}$ lymphocytes and dendritic cells get activated. Type I interferons (IFNs) are produced in the process that help curb the spread of the contagion and also help accelerate the phagocytosis of the viral antigens by macrophages. The adaptive immune system also plays a role in host immunity. B cells are stimulated by the $\mathrm{CD} 4^{+} \mathrm{T}$ cells leading to antibodies specifically targeted against the virus. Viral-infected cells are also directly killed by $\mathrm{CD}^{+} \mathrm{T}$ cells. Proinflammatory cytokines are also produced by the $\mathrm{CD} 8^{+} \mathrm{T}$ cells as part of the immune response [9].

\section{Vaccine development}

The vaccine development stages include the following steps [10]: 1) Research and discovery stage: Conceptualization of the research and conduction of laboratory research by scientists occurs at this stage. 2) Preclinical phase: Testing in animals and additional research is conducted paving the way towards the clinical phase once safety is assessed. 3) Clinical development: All data obtained so far is submitted to the Food and Drug Administration (FDA). After FDA assessment and approval, clinical trials are conducted in three phases. In phase 1 (takes weeks to months), 20 to 100 volunteers are recruited. Information of possible adverse effects and information on efficacy of the vaccine if possible is collected. In phase 2 (can take up to 2 years), hundreds of volunteers are recruited with a diverse group of volunteers for randomized controlled trials. Additional information on safety, side effects, dosage of vaccine and immune response is gathered. In phase 3 (takes from 1 to 4 years), thousands of people are recruited in this phase. Larger sample data collection is conducted. 4) Biologics License Application (BLA) is submitted to the FDA. This includes submission of preclinical, clinical data and details of the manufacturing process and facility information are submitted to the FDA. 5) After approval, prescribing information and 
Table 1. Vaccines Approved in USA and Other Countries for Emergency Use

\begin{tabular}{llll}
\hline Country of development & Developer & Type of vaccine & Usage \\
\hline USA/Germany & Pfizer-BioNTech & mRNA & $\begin{array}{l}\text { Approved in several countries. Emergency use in USA, EU, UK, } \\
\text { Bahrain, Canada, Saudi Arabia, Mexico, etc. } \\
\text { USA }\end{array}$ \\
Russia & Moderna & mRNA & Approved in Switzerland. Emergency use in USA, UK and EU \\
UK/Sweden & Gamaleya & Viral vector & Early use in Russia \\
\hline China & Oxford-AstraZeneca & Viral vector & Emergency use in UK and EU \\
USA/Belgium & CanSino & Viral vector & Approved in China \\
Russia & Johnson and Johnson & Viral vector & Emergency use in USA and Bahrain \\
China & Vector Institute & Protein subunit & Early use in Russia \\
China & Sinopharm & Inactivated virus & Approved in China, UAE and Bahrain. Emergency use in Egypt \\
China & Sinovac & Inactivated virus & Approved in China. Emergency use in Brazil \\
India & Sinopharm-Wuhan & Inactivated virus & Limited use in China and UAE \\
\hline
\end{tabular}

USA: United States of America; UK: United Kingdom; EU: European Union; UAE: United Arab Emirates.

labeling are submitted. Monitoring for safety and effectiveness continues. Vaccines are released by lots with product monitoring through a real-time system set up. COVID-19 being a global public health emergency, vaccine development can be sped up by combining phase 1 and 2 . The vaccines can also be approved more swiftly on an emergent basis and released sooner for usage. The United States FDA (USFDA) has approved Pfizer-BioNTech, Moderna and Janssen COVID-19 vaccines for emergency use. There are other vaccines that have been approved around the world (Table 1).

There are several vaccines being developed for SARS$\mathrm{CoV}-2$. At the time of this paper, there were 81 vaccines in clinical development (Figs. 1-4) and 182 vaccines in preclinical development [11]. Different technologies are being used for the vaccine development [12]. Moderna and Janssen COVID-19 vaccines have been authorized for use in ages 18 years and older. Pfizer-BioNTech COVID-19 vaccine has been authorized for use in ages 16 years and older. Severe allergic reaction (e.g., anaphylaxis) after a prior dose or to any component of the COVID-19 vaccine and an immediate allergic reaction of any given severity to a prior dose or known (diagnosed) allergy to any component of the vaccine are the current contraindications to these vaccines [13].

\section{Messenger RNA (mRNA)-Based Vaccines: Pfiz- er-BioNTech and Moderna}

Pfizer-BioNTech and Moderna developed their vaccines based on mRNA. The mRNA vaccines combine all the important immunological properties with having a balanced humoral and cellular immunological response [14].

\section{Mechanism of action}

Unlike traditional vaccines, a synthetically created RNA sequence of virus fragments encoding the $\mathrm{S}$ protein is injected.
The mRNA fragment is placed in the lipid nanoparticle (LNP) vehicle which prevents degradation by the host until it is taken by the cell [15]. The mRNA strand does not enter the nucleus of the cell. Thus, these vaccines do not affect the genetic makeup of the cells [16]. The intracellular lipases degrade the LNP and expose the mRNA. The dendritic cells take up these fragments by phagocytosis. Then, ribosomes read the mRNA and produce viral antigens which are further broken down by proteasomes. These viral antigens are taken up by major histocompatibility complex (MHC) class I and II molecules further activating the dendritic cells. These activated cells present $\mathrm{T}$ and $\mathrm{B}$ cells $\left(\mathrm{CD}^{+}\right.$cytotoxic $\mathrm{T}$ cells or $\mathrm{CD}^{+}$helper $\mathrm{T}$ cells) in the lymph nodes resulting in stimulation of cellular and humoral immunity [17].

\section{Advantages}

These vaccines have a relatively low cost of production and faster manufacturing time.

\section{Disadvantages}

They can have comparatively lower immunogenicity and more than one dose of vaccine may be required.

\section{Pfizer-BioNTech}

\section{Clinical trials}

The main objective of clinical trials is to determine the safety, tolerability, efficacy and immunogenicity profiles of mRNA vaccines in healthy adults after one or two doses. These studies are multicenter, multinational, randomized, placebo-controlled and observer-blinded [18]. 
30

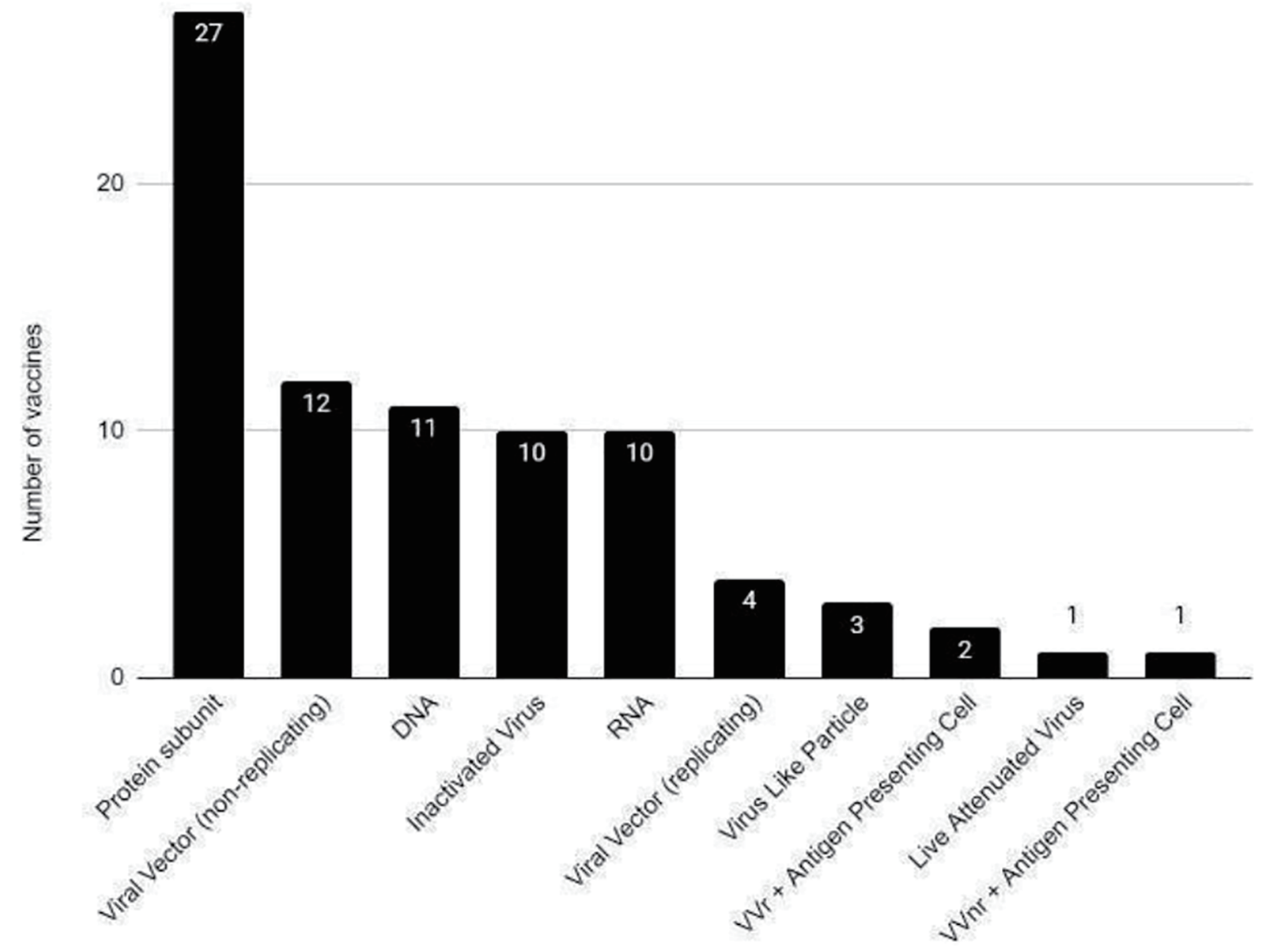

Type of vaccine

Figure 2. COVID-19 vaccines in clinical development phase. COVID-19: coronavirus disease 2019. Reference: Draft landscape of COVID-19 candidate vaccines. World Health Organization. http://www.who.int/publications/m/item/draft-landscape-of-covid19-candidate-vaccines. Accessed January 17, 2021.

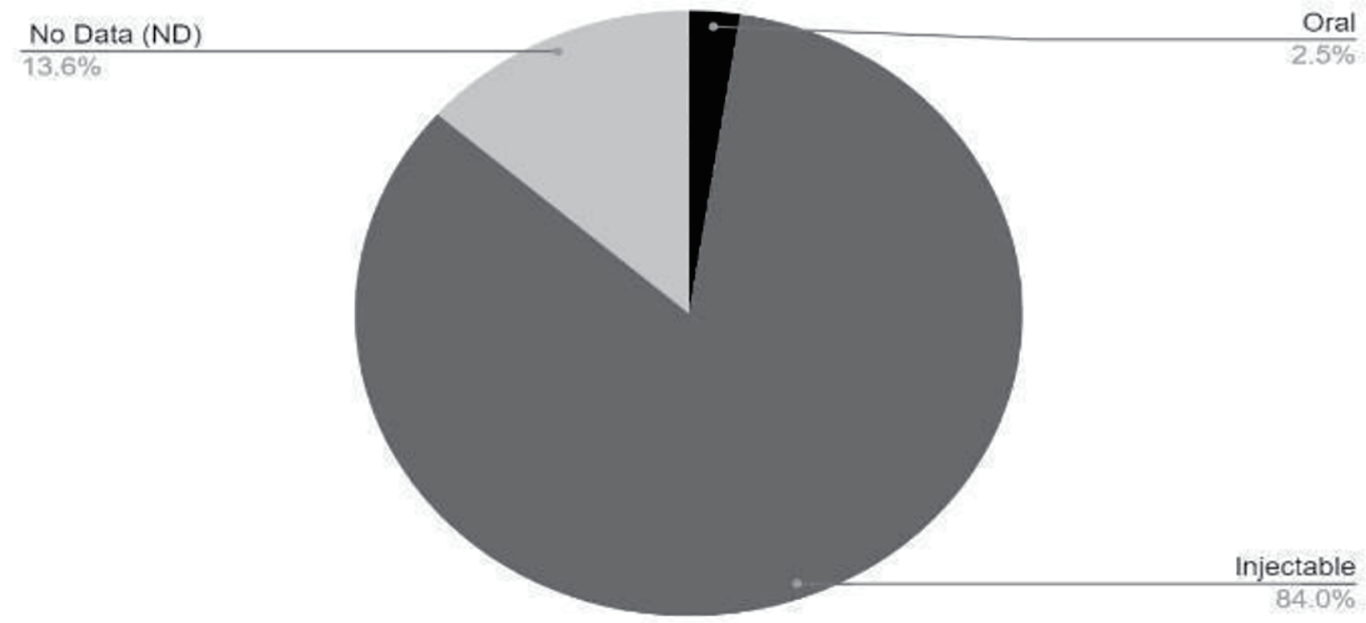

Figure 3. Route of administration of vaccines in clinical development phase. COVID-19: coronavirus disease 2019. Reference: Draft landscape of COVID-19 candidate vaccines. World Health Organization. http://www.who.int/publications/m/item/draft-landscape-of-covid-19-candidate-vaccines. Accessed January 17, 2021. 


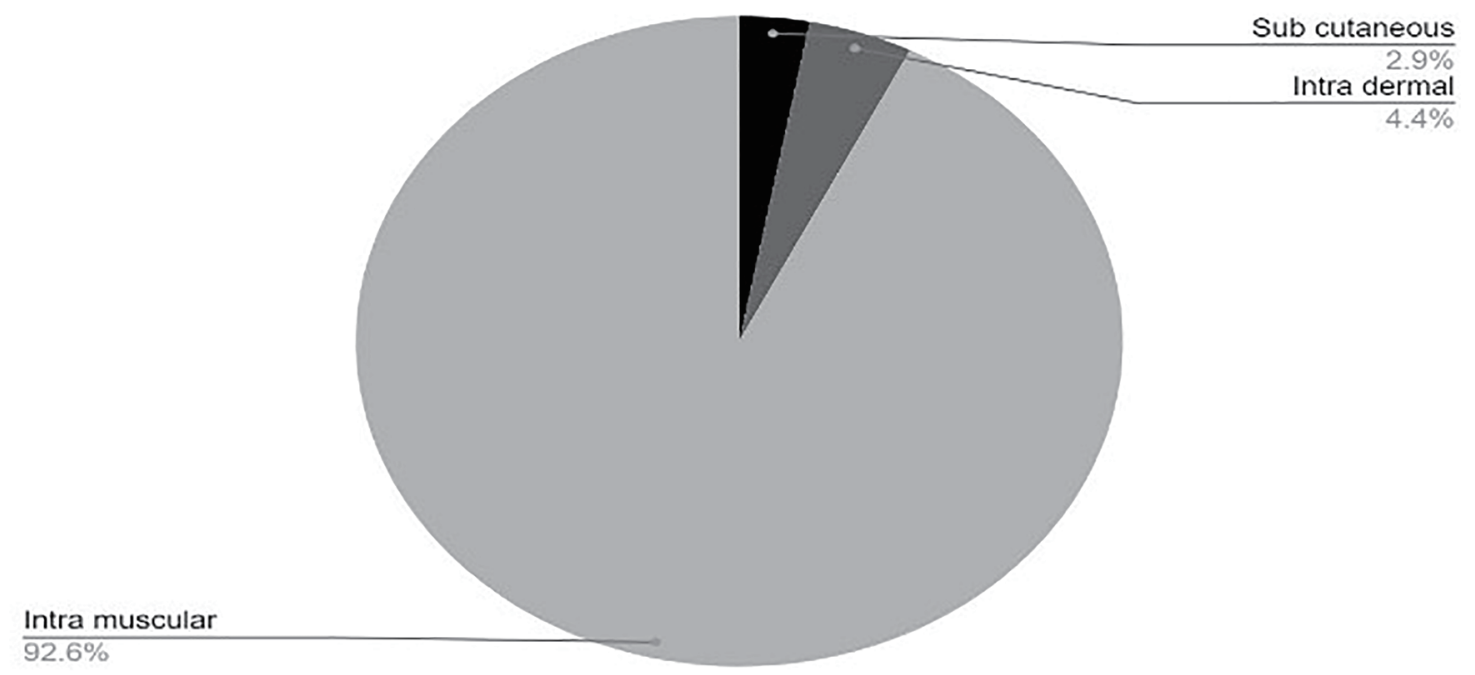

Figure 4. Injectable vaccines in clinical development phase. COVID-19: coronavirus disease 2019. Reference: Draft landscape of COVID-19 candidate vaccines. World Health Organization. http://www.who.int/publications/m/item/draft-landscape-of-covid19-candidate-vaccines. Accessed January 17, 2021.

Phase 1 is mainly to identify the preferred vaccine candidates and doses. Each group in phase 1 has 15 participants. Of them, 12 received an active vaccine, and three received a placebo. A total of 13 groups were studied in two different age groups of $18-55$ and $65-85$ years [17]. A report of analysis of 37 participants in Germany showed a significant immune response against SARS-CoV-2 by developing neutralizing antibodies, TH1 type $\mathrm{CD} 4^{+} \mathrm{T}$ cells, and also $\mathrm{CD} 8^{+} \mathrm{T}$ cells for 85 days. These T-cell responses were directed against the $\mathrm{S}$ protein in various regions [19].

Phases 2 and 3 are to identify the efficacy and tolerability of the vaccine. The vaccine is tested in three different age groups of $12-15,16-55$ and $>55$ years. These phases have enrolled 44,000 participants in total. Of them, 2,259 were 12 - 15 years, 26,181 were 16 - 55 years, and 17,879 were $>55$ years of age [20]. In data from more than 153 clinical trial sites in different countries, the participants have tolerated the vaccine well and also showed the efficacy of $95 \%$ in the $16-55$ years group and $93.8 \%$ in $>55$ years participants in preventing the COVID-19 disease before infection 7 days or more after receiving the second dose [21].

According to a report on February 18, 2021, both com- panies are about to commence the global phase $2 / 3$ trial to evaluate the safety, tolerability and immunogenicity of the COVID-19 vaccine in pregnant women. This is a randomized, placebo-controlled, observer-blinded study that comprises nearly 4,000 healthy pregnant females who are 18 years and older in their 24 - 34 weeks of gestation. Each participant in the study will be monitored for $7-10$ months to assess the safety in infants and also the possibility of transfer of antibodies to their infants. After delivery, each infant will be monitored for 6 months. The same report also mentions the expectations to start the additional studies in children between the ages of 5 and 11 years over the next few months [22].

The USFDA has issued the first emergency use authorization for the Pfizer vaccine on December 11, 2020 for individuals $\geq 16$ years of age, which is being the first vaccine against COVID-19 [23]. The Pfizer-BioNTech is administered intramuscularly in the deltoid region. The vaccine should be given in two doses, 21 days apart.

In a recent report published in NEJM, Pfizer's BNT162b2 illustrated the neutralizing antibodies against the United Kingdom (B.1.1.7), South Africa (B.1.351) and Brazil (P.1) lineage variants [24].

Table 2. Side Effects From Pfizer-BioNTech, Moderna and Janssen COVID-19 Vaccines

\begin{tabular}{lll}
\hline COVID-19 vaccine & Common side effects & Other adverse effects \\
\hline Pfizer-BioNTech & $\begin{array}{l}\text { Fever, cough, fatigue, headache, shortness of breath, } \\
\text { chills, muscle pain, sore throat, diarhea, or vomiting, } \\
\text { local injection site redness or swelling. }\end{array}$ & $\begin{array}{l}\text { Lymphadenopathy, Bell's palsy, } \\
\text { paroxysmal ventricular arrhythmia, right } \\
\text { leg paresthesia and shoulder injury. }\end{array}$ \\
Moderna & $\begin{array}{l}\text { Fever, local pain, swelling, tenderness and erythema at } \\
\text { the injection site. Axillary lymphadenopathy, fatigue, } \\
\text { headache, myalgia, arthralgia, chills and nausea/vomiting. } \\
\text { Manssen }\end{array}$ & $\begin{array}{l}\text { Hypersensitivity reactions, Bell's palsy } \\
\text { and some other lymphadenopathy. }\end{array}$ \\
& $\begin{array}{l}\text { Main, fatigue, headache and myalgia. } \\
\text { paingection site }\end{array}$ & \\
\hline
\end{tabular}

COVID-19: coronavirus disease 2019. 


\section{Storage}

This vaccine is distributed as a multi-dose vial, stored at the temperature of -80 to $-60{ }^{\circ} \mathrm{C}$. After dilution, the vaccine is stored between 2 to $25^{\circ} \mathrm{C}$ and used within $6 \mathrm{~h}$ from dilution [25]. The Pfizer-BioNTech has submitted the data to FDA which demonstrated the stability of this vaccine at temperatures when stored at -25 to $-15^{\circ} \mathrm{C}$ for 2 weeks [26].

\section{Side effects of the vaccine}

These studies reported transient side effects that are commonly seen in participants who received BNT162b2 than placebo. The commonly reported events after 2 months of follow-up were fever, cough, fatigue, headache, shortness of breath, chills, muscle pain, sore throat, diarrhea, or vomiting. A large number of participants showed local injection site redness or swelling mostly after the second dose which cured in $1-2$ days. Other severe adverse events were reported following the injection of the vaccine such as $0.3 \%$ of participants reported lymphadenopathy, four patients with Bell's palsy, paroxysmal ventricular arrhythmia, right leg paresthesia and shoulder injury. Safety monitoring for the participants enrolled in clinical trials will be continued for 2 years after the administration of the second dose of vaccine [27] (Table 2).

\section{Moderna}

This is a nucleoside-modified mRNA vaccine that induces an immune response to $\mathrm{S}$ antigen to protect against COVID-19.

\section{Phase 1 trial}

The main objective of phase 1 clinical trial is to determine the safety and reactogenicity of the vaccine. A total of 120 healthy males and non-pregnant females, aged more than 18 years, were enrolled in phase 1 clinical trials. All the participants were divided into 13 groups and given five different doses starting from 10, 25, 50, 100 and $250 \mu \mathrm{g}$ on days 1 and 29 [28]. The data from this study suggest that the mRNA-1273 vaccine is safe and well-tolerated, showing neutralizing antibody titer levels in all participants starting at a dose as low as $25 \mu \mathrm{g}$ [29]. The participants exhibited the highest levels of antibodies, 119 days following the first dose and 90 days of the second dose.

\section{Phases 2/3 trials}

Phases $2 / 3$ consist of participants aged 18 years and older. The randomization was made with age and health risk. A total of 30,420 participants were enrolled in phase 3 . Half of them received the Moderna COVID-19 vaccine and another half received a saline placebo. These participants will be followed for 24 months after the second dose to study safety and efficacy. According to phase 3 trial data, it is found to have $94.1 \%$ ef- ficacy after the second dose [30].

In a study by Moderna published in the New England Journal of Medicine, their mRNA vaccine has demonstrated the effectiveness against the UK (B.1.1.7), South Africa (B.1.351) and 20E (EU1), 20A.EU2, N439K-D614G and the mink cluster 5 variant strains [31].

USFDA authorized the Moderna mRNA vaccine for its emergency use on December 18, 2020. Like Pfizer, the Moderna vaccine is administered intramuscularly. A $0.5 \mathrm{~mL}$ vaccine consists of $100 \mu \mathrm{g}$ of nucleoside-modified mRNA and is given to the individual in two doses, 28 days apart and this vaccine is not approved yet for the pediatric age group.

\section{Storage}

This is stored and distributed in multi-dose vials at -25 to -15 ${ }^{\circ} \mathrm{C}[30]$.

\section{Side effects of the vaccine}

The common adverse reactions in clinical trials after the Moderna COVID-19 vaccine were fever, local pain (92\%), swelling (14.7\%), tenderness, erythema at the injection site (10\%), axillary lymphadenopathy $(19.8 \%)$, fatigue $(70 \%)$, headache $(64.7 \%)$, myalgia (61.5\%), arthralgia (46.4\%), chills (45.4\%) and nausea/ vomiting $(23 \%)$. Other uncommon adverse reactions included hypersensitivity reactions (1.5\%), Bell's palsy (three patients) and some other lymphadenopathy-related events [30] (Table 2).

\section{Viral Vector-Based Vaccines}

Adenoviruses have this unique property to act as vaccine vectors and induce both innate and adaptive immune response in mammalian hosts [32]. Adenoviruses are double-stranded deoxyribonucleic acid (DNA) viruses and were discovered by Rowe and his colleagues in 1953 from human adenoid tissue [33].

\section{Mechanism of action of adenovirus as vaccine vector}

The deletion of E1 genes terminates the viral replication. The compact genomic structure of adenovirus makes it a suitable option for the incorporation of a foreign DNA. Genes encoding the SARS-CoV-2 S protein are isolated and following gene sequencing, they are introduced into the adenovirus vector. These viruses after growing in specialized cells are developed into vaccines, which upon injecting into the host, trigger humoral and cellular immune response, resulting in destruction and neutralizing virus on future exposure with an actual SARS-CoV-2 virus [34].

\section{Advantages}

These vaccines have a relatively fast manufacturing time. 


\section{Disadvantages}

The efficacy of the vaccine is variable based on the host's immune response to the viral vector.

\section{Janssen}

JNJ-78436735 or Ad26.COV2.S is an adenoviral vector-based vaccine against SARS-CoV-2 developed by Janssen Phaemaceutica of Johnson \& Johnson in collaboration with Beth Israel Deaconess Medical Center.

\section{Mechanism of action}

The gene encoding the coronavirus $\mathrm{S}$ protein is added to the replication deficient adenovirus 26 . The genetically engineered adenovirus 26 is developed into vaccine. The vaccine once injected into the host cell is engulfed by the cell. Once inside the cell's nucleus, the $\mathrm{S}$ protein gene is copied into mRNA. The mRNA leaves the nucleus and starts building $\mathrm{S}$ proteins, resulting in activation of immune response. Humoral and cell-mediated immune responses are activated after the first dose. Memory B cells and memory T cells result in longterm immune response which later fights against the virus on exposure.

\section{Preclinical studies}

Preclinical studies done on non-human primates to show the protective efficacy and immunogenicity of a single dose of Ad26 vaccine were published in Nature. Fifty-two rhesus macaques were immunized with Ad26 vaccine vector and then exposed to SARS-CoV-2 by the intranasal and intratracheal routes. Broncho alveolar and nasal swab after SARS-CoV-2 challenge proved complete or near complete protection by induction of neutralizing antibodies against the virus [35].

\section{Phases 1 and 2 studies}

Phases 1 and 2 are to assess the safety, reactogenicity and immunogenicity of Janssen's vaccine in adult volunteers at two dose levels. The positive interim results after a single dose were supportive of further development of the vaccine. Vaccine was administered intramuscularly as single-dose or twodose schedules, 8 weeks apart, in healthy adults aged 18 - 55 years and greater than 65 years. Healthy volunteers received Ad26.COV2.S at a dose level of $5 \times 10^{10}$ or $1 \times 10^{11}$ viral particles (vp) either as a single-dose or as a two-dose schedule spaced by 56 days. Volunteers were grouped into three cohorts: 18 - 55 years old (cohorts $1 \mathrm{a}$ and $1 \mathrm{~b} ; \mathrm{n}=402$ ) and healthy elderly older than 65 years old (cohort $3 ; n=394$ ). Antibody levels were measured by enzyme-linked immunosorbent assay (ELISA) and neutralizing titers were measured in a wild-type virus neutralization assay (wtVNA). CD4 ${ }^{+}$T-helper (Th) 1 and
Th2, and $\mathrm{CD}^{+}$immune responses were assessed by intracellular cytokine staining (ICS).

Results after single dose showed seroconversion rate in wtVNA (50\% inhibitory concentration (IC50)) at day 29 after immunization in cohort 1 a already reached $92 \%$ with geometric mean titer (GMTs) of 214 (95\% confidence interval (CI): $177-259)$ and $92 \%$ with GMTs of 243 (95\% CI: 200 - 295) for the $5 \times 10^{10}$ and $1 \times 10^{11}$ vp dose levels, respectively. A similar immunogenicity profile was observed in the first 15 participants in cohort 3, where $100 \%$ seroconversion (6/6) (GMTs of 196 (95\% CI: 69 - 560)) and 83\% seroconversion (5/6) (GMTs of $127(95 \% \mathrm{CI}:<58-327))$ were observed for the $5 \times 10^{10}$ or $1 \times 10^{11}$ vp dose level, respectively. Seroconversion for S antibodies as measured by ELISA (U/mL) was observed in $99 \%$ of cohort 1a participants (GMTs of 528 (95\% CI: $442-630)$ and 695 (95\% CI: $596-810)$ ), for the $5 \times 10^{10}$ or $1 \times 10^{11} \mathrm{vp}$ dose level, respectively, and in 100\% (6/6 for both dose levels) of cohort 3 with GMTs of 507 (95\% CI: 181 - 1,418) and 248 (95\% CI: 122 - 506), respectively. On day 14 after immunization, Th1 cytokine producing S-specific $\mathrm{CD}^{+} \mathrm{T}$-cell responses was measured in $80 \%$ and $83 \%$ of participants in cohort $1 \mathrm{a}$ and 3 , respectively. $\mathrm{CD}^{+} \mathrm{T}$-cell responses were also prominent in both cohort 1a and 3, for both dose levels. The study is ongoing at different clinical sites in the USA and Belgium. The trial is registered at ClinicalTrials.gov, NCT04436276 [36].

\section{Phase 3}

The phase 3 ENSEMBLE study is an ongoing study estimated to enroll up to 60,000 participants of 18 years or older worldwide. The purpose of the study is to assess the safety, side effect and immunogenicity of the single-dose regimen of JNJ-78436735, in a wider range of population to evaluate the long-term efficiency of the vaccine. Participants will receive intramuscular (IM) injection of Ad26.COV2.S at a dose level of $5 \times 10^{10} \mathrm{vp}$ as single-dose vaccine on day 1 compared to IM injection of placebo on day 1 in the comparator group. The trial is registered in ClinicalTrials.gov, NCT04505722 [37, 38].

Parallel to ENSEMBLE, an ENSEMBLE 2 trial is ongoing to assess the efficacy, safety and immunogenicity of two doses of vaccine regimen versus placebo in adults of 18 years old and older. The study will enroll up to 30,000 participants being conducted in collaboration with the UK National Institute for Health Research (NIHR). The trial is registered in ClinicalTrials.gov NCT04614948. Both trials are randomized, double-blinded, placebo-controlled [37-39]. Janssen will aim to enroll participants in Belgium, Colombia, France, Germany, Philippines, South Africa, Spain, United Kingdom and the USA. In order to evaluate the efficacy of Janssen's COVID-19 vaccine candidate, clinical trial sites in countries and areas with high incidence of COVID-19 and the ability to achieve a rapid initiation were selected.

\section{Storage}

The Johnson \& Johnson vaccine can be refrigerated for up to 
3 months at $36-46^{\circ} \mathrm{F}\left(2-8^{\circ} \mathrm{C}\right)$ and up to 2 years at $-20^{\circ} \mathrm{C}$.

Side effects of the vaccine

Mild to moderate febrile episodes occurred and resolved within 1 to 2 days after vaccination. The most frequent local adverse event was injection site pain, fatigue, headache and myalgia [36] (Table 2).

\section{Conclusion}

Vaccines are an essential tool in our battle against COVID-19. Some of the COVID-19 vaccines have completed their phase 3 trials, while many other potential vaccines are still in developmental stages. Till date, COVID-19 has claimed over 532,000 lives in the USA alone and over 29 million people were infected with the virus. Over 119 million people were infected worldwide and unfortunately 2.64 million people have lost their lives to this deadly virus within a span of 13 months [40]. While the full scale impact of the pandemic is yet to be known in years to come, the magnitude of the pandemic has catapulted us into an accelerated learning curve, research and development including involving testing, treatment, prevention and vaccination. Vaccines have proven to be a tested tool in being the most effective way to prevent infectious diseases. Vaccines have helped effectively eradicate diseases like smallpox and have helped drastically reduce the incidence of diseases like polio, measles, pertussis, mumps, to name a few. While it used to take close to a decade for a vaccine to be developed and undergo rigorous testing until its production and availability to the public, but over the past year, we have seen multiple vaccines in different phases of testing against SARSCoV-2 virus. So far three vaccines have received emergency use authorization in the USA. The mRNA vaccines have used a novel technology and first of their kind promising alternative to conventional vaccine approaches because of their hypodensity and capacity for rapid development and potential for low cost manufacture and safe administration. These vaccines give us a hope to return to a new normalcy.

\section{Acknowledgments}

None to declare.

\section{Financial Disclosure}

The authors declare that they do not have a financial relationship with any commercial entity that has an interest in the subject of this manuscript.

\section{Conflict of Interest}

The authors declare that they do not have a conflict of interest.

\section{Author Contributions}

DV, PA, SVM, RV and GM have performed literature search, drafted the manuscript and did critical editing. DV has contributed to the illustrations. DV and PA have carefully supervised this manuscript preparation and writing.

\section{Data Availability}

The authors declare that data supporting the findings of this study are available within the article.

\section{Abbreviations}

RNA: ribonucleic acid; S: spike; M: matrix; N: nucleocapsid; E: envelope; FDA: Food and Drug Administration; mRNA: messenger ribonucleic acid; SARS-CoV-2: severe acute respiratory syndrome coronavirus 2 ; IgG: immunoglobulin $\mathrm{G}$; ACE2: angiotensin-converting enzyme 2; IFN: interferon; BLA: Biologics License Application; LNP: lipid nanoparticle; USFDA: United States Food and Drug Administration; DNA: deoxyribonucleic acid; wtVNA: wild-type virus neutralization assay; ICS: intracellular cytokine staining; GMT: geometric mean titer; ELISA: enzyme-linked immunosorbent assay; IM: intramuscular; NIHR: National Institute for Health Research

\section{References}

1. Guo YR, Cao QD, Hong ZS, Tan YY, Chen SD, Jin HJ, Tan KS, et al. The origin, transmission and clinical therapies on coronavirus disease 2019 (COVID-19) outbreak - an update on the status. Mil Med Res. 2020;7(1):11.

2. Zhu N, Zhang D, Wang W, Li X, Yang B, Song J, Zhao X, et al. A novel coronavirus from patients with pneumonia in China, 2019. N Engl J Med. 2020;382(8):727-733.

3. Fehr AR, Perlman S. Coronaviruses: an overview of their replication and pathogenesis. Methods Mol Biol. 2015;1282:1-23.

4. McBride R, van Zyl M, Fielding BC. The coronavirus nucleocapsid is a multifunctional protein. Viruses. 2014; 6(8):2991-3018.

5. McClenaghan C, Hanson A, Lee SJ, Nichols CG. Coronavirus Proteins as Ion Channels: Current and Potential Research. Front Immunol. 2020;11:573339.

6. Thomas S. The structure of the membrane protein of SARS-CoV-2 resembles the sugar transporter SemiSWEET. Pathog Immun. 2020;5(1):342-363.

7. Kirchdoerfer RN, Cottrell CA, Wang N, Pallesen J, Yassine HM, Turner HL, Corbett KS, et al. Pre-fusion structure of a human coronavirus spike protein. Nature. 2016;531(7592):118-121.

8. Yesudhas D, Srivastava A, Gromiha MM. COVID-19 outbreak: history, mechanism, transmission, structural studies and therapeutics. Infection. 2021;49(2):199-213.

9. Mortaz E, Tabarsi P, Varahram M, Folkerts G, Adcock 
IM. The immune response and immunopathology of COVID-19. Front Immunol. 2020;11:2037.

10. Center for biologics evaluation and Research. Vaccine Development - 101. U.S. Food and Drug Administration. https://www.fda.gov/vaccines-blood-biologics/development-approval-process-cber/vaccine-development-101. Accessed February 5, 2021.

11. Draft landscape of COVID-19 candidate vaccines. World Health Organization. https:/www.who.int/publications/ $\mathrm{m} /$ item/draft-landscape-of-covid-19-candidate-vaccines. Accessed January 17, 2021.

12. Understanding how COVID-19 vaccines work. Centers for Disease Control and Prevention. https://www.cdc.gov/ coronavirus/2019-ncov/vaccines/different-vaccines/ how-they-work.html?CDC_AA_refVal=https $\% 3 \mathrm{~A}$ $\% 2 \mathrm{~F} \% 2 \mathrm{Fwww} . \mathrm{cdc}$.gov\%2Fcoronavirus $\% 2 \mathrm{~F} 2019$ ncov $\% 2$ Fvaccines $\% 2$ Fabout-vaccines $\% 2$ Fhow-theywork.html. Accessed January 17, 2021.

13. Interim clinical considerations for use of covid-19 vaccines. 2021. Retrieved March 30, 2021. https://www.cdc. gov/vaccines/covid-19/info-by-product/clinical-considerations.html\#Contraindications.

14. Schlake T, Thess A, Fotin-Mleczek M, Kallen KJ. Developing mRNA-vaccine technologies. RNA Biol. 2012;9(11): 1319-1330.

15. Batty CJ, Heise MT, Bachelder EM, Ainslie KM. Vaccine formulations in clinical development for the prevention of severe acute respiratory syndrome coronavirus 2 infection. Adv Drug Deliv Rev. 2021;169:168-189.

16. Understanding and Explaining mRNA COVID-19 Vaccines. Centers for Disease Control and Prevention. https://www.cdc.gov/vaccines/covid-19/hcp/mrna-vaccinebasics.html. Published November 24, 2020.

17. Pardi N, Hogan MJ, Porter FW, Weissman D. mRNA vaccines - a new era in vaccinology. Nat Rev Drug Discov. 2018;17(4):261-279.

18. A phase $1 / 2 / 3$, placebo-controlled, randomized, observerblind, dose-finding study to evaluate the safety, tolerability, immunogenicity, and efficacy of sars-cov-2 RNA vaccine candidates against COVID-19 in healthy individuals. n.d.). Retrieved from: https://pfe-pfizercom-d8-prod.s3. amazonaws.com/2020-11/C4591001_Clinical_Protocol_ Nov2020.pdf.

19. Pfizer and BioNTech provide data from German phase $1 / 2$ study further characterizing immune response following immunization with lead COVID-19 vaccine candidate BNT162b2. Pfizer. https://www.pfizer.com/news/ press-release/press-release-detail/pfizer-and-biontechprovide-data-german-phase-12-study. Accessed February 25, 2021.

20. BioNTech COVID-19 vaccine trial overview. Pfizer. https://www.pfizer.com/science/coronavirus/vaccine. Accessed February 25, 2021.

21. Pfizer and BioNTech announce publication of results from landmark phase 3 trial of BNT162b2 COVID-19 vaccine candidate. New England Journal of Medicine. Pfizer. https://www.pfizer.com/news/press-release/pressrelease-detail/pfizer-and-biontech-announce-publicationresults-landmark. Accessed February 25, 2021.
22. Pfizer and BioNTech commence global clinical trial to evaluate COVID-19 vaccine in pregnant women. Pfizer. https://www.pfizer.com/news/press-release/press-release-detail/pfizer-and-biontech-commence-global-clinical-trial-evaluate. Accessed February 25, 2021.

23. Commissioner of the Pfizer-BioNTech COVID-19 vaccine. U.S. Food and Drug Administration. https://www. fda.gov/emergency-preparedness-and-response/coronavirus-disease-2019-covid-19/pfizer-biontech-covid19-vaccine. Accessed February 25, 2021.

24. Kadire SR, Polack FP. Neutralizing activity of BNT162b2-elicited serum - preliminary report: NEJM. New England Journal of Medicine. https://www.nejm. org/doi/10.1056/NEJMc2102017?url_ver=Z39.882003\&rfr_id=ori\%3Arid\%3Acrossref.org \&rfr_dat $=\mathrm{cr}$ pub++0pūbmed. Published February 17, 2021. $\bar{A} c c e s s e \bar{d}$ February 25, 2021.

25. U.S. Food and Drug Administration. FDA. https://www. fda.gov/media/144245. Accessed February 25, 2021.

26. Pfizer and BioNTech Submit COVID-19 vaccine stability data at Standard Freezer Temperature to the U.S. FDA. Pfizer. https://www.pfizer.com/news/press-release/pressrelease-detail/pfizer-and-biontech-submit-covid-19-vaccine-stability-data. Accessed February 25, 2021.

27. Polack FP, Thomas SJ, Kitchin N, Absalon J, Gurtman A, Lockhart S, Perez JL, et al. Safety and efficacy of the BNT162b2 mRNA COVID-19 vaccine. N Engl J Med. 2020;383(27):2603-2615.

28. Safety and immunogenicity study of 2019-nCoV Vaccine (mRNA-1273) for prophylaxis of SARS-CoV-2 infection (COVID-19) - Full Text View. Full Text View - ClinicalTrials.gov. https://clinicaltrials.gov/ct2/show/ NCT04283461. Accessed February 25, 2021.

29. Moderna announces positive interim phase 1 data for its mRNA vaccine (mRNA-1273) against novel coronavirus. Moderna, Inc. https://investors.modernatx.com/newsreleases/news-release-details/moderna-announces-positive-interim-phase-1-data-its-mrna-vaccine. Accessed February $25,2021$.

30. U.S. Food and Drug Administration. FDA. https://www. fda.gov/media/144637. Accessed February 25, 2021.

31. Kadire SR, Polack FP. Serum neutralizing activity elicited by mRNA-1273 vaccine - preliminary report: NEJM. New England Journal of Medicine. https://www.nejm. org/doi/full/10.1056/NEJMc2102179?query=featured_ home. Published February 17, 2021. Accessed February 25, 2021.

32. Tatsis N, Ertl H. Adenoviruses as vaccine vectors. Europe PMC. http://europepmc.org/abstract/MED/15451446. Published 2004. Accessed February 13, 2021.

33. Ghebremedhin B. Human adenovirus: Viral pathogen with increasing importance. Eur J Microbiol Immunol (Bp). 2014;4(1):26-33.

34. Feng L, Wang Q, Shan C, Yang C, Feng Y, Wu J, Liu X, et al. An adenovirus-vectored COVID-19 vaccine confers protection from SARS-COV-2 challenge in rhesus macaques. Nat Commun. 2020;11(1):4207.

35. Mercado NB, Zahn R, Wegmann F, Loos C, Chandrashekar A, Yu J, Liu J, et al. Single-shot Ad26 vaccine 
protects against SARS-CoV-2 in rhesus macaques. Nature. 2020;586(7830):583-588.

36. Sadoff J, Gars ML, Shukarev G, Heerwegh D, Truyers C, de Groot AM, Stoop J, et al. Safety and immunogenicity of the Ad26.COV2.S COVID-19 vaccine candidate: interim results of a phase $1 / 2 \mathrm{a}$, double-blind, randomized, placebo-controlled trial. medRxiv. 2020.

37. Johnson \& Johnson. Content Lab U.S. https://www.jnj. com/johnson-johnson-initiates-second-global-phase3-clinical-trial-of-its-janssen-covid-19-vaccine-candidate. Accessed March 4, 2021.

38. A study of Ad26.COV2.S for the prevention of SARS-
CoV-2-mediated COVID-19 in adult participants (ENSEMBLE). A study of Ad26.COV2.S for the prevention of SARS-CoV-2-mediated COVID-19 in adult participants - Full Text View - ClinicalTrials.gov.

39. A study of Ad26.COV2.S for the prevention of SARSCoV-2-mediated COVID-19 in adults (ENSEMBLE 2). A study of Ad26.COV2.S for the prevention of SARSCoV-2-mediated COVID-19 in adults - Full Text View ClinicalTrials.gov.

40. CDC COVIDData Tracker. n.d.). Retrieved March 15,2021. https://covid.cdc.gov/covid-data-tracker/\#datatrackerhome. 